\title{
Article
}

\section{Qualitative analysis of solutions for a parabolic type Kirchhoff equation with logarithmic nonlinearity}

\section{Erhan Pişkin $^{1, *}$ and Tuğrul Cömert ${ }^{1}$}

1 Department of Mathematics, Dicle University, 21280 Diyarbakır, Turkey.

* Correspondence: episkin@dicle.edu.tr

Academic Editor: Aisha Javed

Received: 2 February 2021; Accepted: 21 March 2021; Published: 27 May 2021.

Abstract: In this work, we investigate the initial boundary-value problem for a parabolic type Kirchhoff equation with logarithmic nonlinearity. We get the existence of global weak solution, by the potential wells method and energy method. Also, we get results of the decay and finite time blow up of the weak solutions.

Keywords: Blow up; Global existence; Logarithmic nonlinearity; Parabolic type Kirchhoff equation.

MSC: 35A01; 35B40; 35K20.

\section{Introduction}

$\mathbf{I}^{\mathrm{n}}$

n this work, we investigate the existence of global, decay and finite time blow up of solutions for the parabolic type Kirchhoff equation with logarithmic source term

$$
\begin{cases}u_{t}-M\left(\|\nabla u\|^{2}\right) \Delta u-\Delta u_{t}=u^{k-1} \ln |u|-\oint_{\Omega} u^{k-1} \ln |u| d x, & x \in \Omega, t>0, \\ u(x, t)=0, & x \in \partial \Omega, t>0, \\ u(x, 0)=u_{0}(x), & x \in \Omega,\end{cases}
$$

where $\Omega$ is a bound domain in $\mathbb{R}^{n}(n \geq 1)$ with smooth boundary $\partial \Omega$. Also, $M(s)=1+s^{\gamma},(\gamma>0), \oint_{\Omega} u_{0} d x=$ $\frac{1}{|\Omega|} \int_{\Omega} u_{0} d x=0$ and

$$
\begin{cases}2 \gamma+2 \leq k \leq+\infty, & n=1,2 \\ 2 \gamma+2 \leq k \leq \frac{2 n}{n-2}, & n \geq 3\end{cases}
$$

Many other authors studied the problem (1) in a more general form

$$
\begin{cases}u_{t}-\Delta u=f(u)-\oint_{\Omega} f(u) d x, & x \in \Omega, t>0 \\ \frac{\partial u(x, t)}{\partial \eta}=0, & x \in \partial \Omega, t>0 \\ u(x, 0)=u_{0}(x), & x \in \Omega\end{cases}
$$

where $\Omega$ is a bound domain in $\mathbb{R}^{n}(n \geq 1)$ with $|\Omega|$ denoting its Lebesgue measure, the function $f(u)$ is usually taken to be a power of $u$, and $n$ is the outer normal vector of $\partial \Omega$. Studies of logarithmic nonlinearity have a long history in physics as it occurs naturally in different areas of physics such as supersymmetric field theories, optics, quantum mechanics and inflationary cosmology [1,2]. When $M \equiv 1$ and $k=2$, (1) become semilinear pseudo-parabolic equation as follow

$$
u_{t}-\Delta u-\Delta u_{t}=u \log |u| .
$$

Chen and Tian [3] obtained the global existence, behavior of vacuum isolation and blow-up of solutions at $+\infty$ of the Equation (2). Without $\Delta u$, (2) become the following semilinear parabolic equation

$$
u_{t}-\Delta u_{t}=u \log |u|
$$


Chen et al., [4] studied the global existence, decay and blow-up at $+\infty$ of solutions of the Equation (3). Yan and Yang [5] studied nonlocal parabolic equation with logarithmic nonlinearity

$$
u_{t}-\Delta u_{t}=u \log |u|-\oint_{\Omega} u \log |u| d x
$$

Recently, they obtained the results under appropriate conditions on blow-up and existion of the solutions. Toualbia et al., [6] studied the initial boundary value problem of a nonlocal parabolic equation with logarithmic nonlinearity

$$
u_{t}-\operatorname{div}\left(|\nabla u|^{p-2} \nabla u\right)=|u|^{p-2} u \log |u|-\oint_{\Omega}|u|^{p-2} u \log |u| d x .
$$

They obtained the decay, blow up and nonextinction of solutions under appropriate condition. Also, recently some authors studied the parabolic and hyperbolic type equation with logarithmic source term (see [7-15]).

The organization of the remaining part of this paper is as follows: In the next Section 2, we introduce some lemmas which will be needed later. In Section 3, under some conditions, we get the unique global weak solution of the problem (1). Moreover, we find that the decay of solutions. In the lastly, we get the blow up theorem.

\section{Preliminaries}

Throughout this work, we adopt the following abbreviations

$$
\|u\|_{s}=\|u\|_{L^{s}(\Omega)},\|u\|_{H_{0}^{1}(\Omega)}=\left(\|\nabla u\|^{2}+\|u\|^{2}\right)^{\frac{1}{2}}
$$

for $1<s<\infty$.

The energy functional $J$ and Nehari functional $I$ defined on $H_{0}^{1}(\Omega) \backslash\{0\}$ as follow

$$
J(u)=\frac{1}{2}\|\nabla u\|^{2}+\frac{1}{2(\gamma+1)}\|\nabla u\|^{2(\gamma+1)}-\frac{1}{k} \int_{\Omega}|u|^{k} \ln |u| d x+\frac{1}{k^{2}} \int_{\Omega}|u|^{k} d x,
$$

and

$$
I(u)=\|\nabla u\|^{2}+\|\nabla u\|^{2(\gamma+1)}-\int_{\Omega}|u|^{k} \ln |u| d x .
$$

By (4) and (5), we obtain

$$
J(u)=\frac{1}{k} I(u)+\frac{k-2}{2 k}\|\nabla u\|^{2}+\frac{k-2 \gamma-2}{2 k(\gamma+1)}\|\nabla u\|^{2(\gamma+1)}+\frac{1}{k^{2}} \int_{\Omega}|u|^{k} d x .
$$

Let

$$
\mathcal{N}=\left\{u \in H_{0}^{1}(\Omega) \backslash\{0\}: I(u)=0\right\},
$$

be the Nehari manifold. Thus, we may define

$$
d=\inf _{u \in \mathcal{N}} J(u)
$$

$d$ is positive and is obtained by some $u \in \mathcal{N}$.

Lemma 1. Let $u \in H_{0}^{1}(\Omega) \backslash\{0\}$, we consider the function $j: \lambda \rightarrow J(\lambda u)$ for $\lambda>0$. Then we possess

(i) $\lim _{\lambda \rightarrow 0^{+}} j(\lambda)=0$ and $\lim _{\lambda \rightarrow+\infty} j(\lambda)=-\infty$;

(ii) there is a unique $\lambda^{*}>0$ such that $j^{\prime}\left(\lambda^{*}\right)=0$;

(iii) $j(\lambda)$ is strictly increasing on $\left(0, \lambda^{*}\right)$, strictly decreasing on $\left(\lambda^{*},+\infty\right)$ and takes the maximum at $\lambda^{*} ; I(\lambda u)=$ $\lambda j^{\prime}(\lambda)$ and

$$
I(\lambda u)\left\{\begin{array}{l}
>0, \quad 0<\lambda<\lambda^{*} \\
=0, \quad \lambda=\lambda^{*} \\
<0, \quad \lambda^{*}<\lambda<+\infty
\end{array}\right.
$$


Proof. For $u \in H_{0}^{1}(\Omega) \backslash\{0\}$, by the definition of $j$, we get

$$
\begin{aligned}
j(\lambda) & =\frac{1}{2}\|\nabla(\lambda u)\|^{2}+\frac{1}{2(\gamma+1)}\|\nabla(\lambda u)\|^{2(\gamma+1)}-\frac{1}{k} \int_{\Omega}|\lambda u|^{k} \ln |\lambda u| d x+\frac{1}{k^{2}} \int_{\Omega}|\lambda u|^{k} d x \\
& =\frac{\lambda^{2}}{2}\|\nabla u\|^{2}+\frac{\lambda^{2(\gamma+1)}}{2(\gamma+1)}\|\nabla u\|^{2(\gamma+1)}-\frac{\lambda^{k}}{k} \int_{\Omega}|u|^{k} \ln |u| d x-\frac{\lambda^{k}}{k} \int_{\Omega}|u|^{k} \ln \lambda d x+\frac{\lambda^{k}}{k^{2}} \int_{\Omega}|u|^{k} d x .
\end{aligned}
$$

It is clear that (i) holds due to $\int_{\Omega}|u|^{k} d x \neq 0$. We get

$$
\begin{aligned}
\frac{d}{d \lambda} j(\lambda)= & \lambda\|\nabla u\|^{2}+\lambda^{2 \gamma+1}\|\nabla u\|^{2(\gamma+1)}-\lambda^{k-1} \int_{\Omega}|u|^{k} \ln |u| d x \\
& -\lambda^{k-1} \ln \lambda \int_{\Omega}|u|^{k} d x-\frac{\lambda^{k-1}}{k} \int_{\Omega}|u|^{k} d x+\frac{\lambda^{k-1}}{k} \int_{\Omega}|u|^{k} d x \\
= & \lambda\|\nabla u\|^{2}+\lambda^{2 \gamma+1}\|\nabla u\|^{2(\gamma+1)}-\lambda^{k-1} \int_{\Omega}|u|^{k} \ln |u| d x-\lambda^{k-1} \ln \lambda \int_{\Omega}|u|^{k} d x \\
= & \lambda\left(\|\nabla u\|^{2}+\lambda^{2 \gamma}\|\nabla u\|^{2(\gamma+1)}-\lambda^{k-2} \int_{\Omega}|u|^{k} \ln |u| d x-\lambda^{k-2} \ln \lambda \int_{\Omega}|u|^{k} d x\right) .
\end{aligned}
$$

Let

$$
\varphi(\lambda):=\lambda^{2 \gamma}\|\nabla u\|^{2(\gamma+1)}-\lambda^{k-2} \int_{\Omega}|u|^{k} \ln |u| d x-\lambda^{k-2} \ln \lambda \int_{\Omega}|u|^{k} d x .
$$

Then from $2 \gamma+2 \leq k$ we can conclude that $\lim _{\lambda \rightarrow \infty} \varphi(\lambda)=-\infty, \varphi(\lambda)$ is monotone decreasing when $\lambda>\lambda^{*}$ and there exists a unique $\lambda^{*}$ such that $\left.\varphi(\lambda)\right|_{\lambda=\lambda^{*}=0}$. Hence, we obtain there is a $\lambda^{*}>0$ such that $\|\nabla u\|^{2}+$ $\varphi(\lambda)=0$, which means $\left.\frac{d}{d \lambda} J(\lambda u)\right|_{\lambda=\lambda^{*}=0}$. The conclusion (iii) directly follows from the proof of the conclusion (ii) and

$$
\begin{aligned}
I(\lambda u) & =\|\nabla(\lambda u)\|^{2}+\|\nabla(\lambda u)\|^{2(\gamma+1)}-\int_{\Omega}|\lambda u|^{k} \ln |\lambda u| d x \\
& =\lambda^{2}\|\nabla u\|^{2}+\lambda^{2(\gamma+1)}\|\nabla u\|^{2(\gamma+1)}-\lambda^{k} \int_{\Omega}|u|^{k} \ln |u| d x-\lambda^{k} \int_{\Omega}|u|^{k} \ln \lambda d x \\
& =\lambda^{2}\|\nabla u\|^{2}+\lambda^{2(\gamma+1)}\|\nabla u\|^{2(\gamma+1)}-\lambda^{k} \int_{\Omega}|u|^{k} \ln |u| d x-\lambda^{k} \ln \lambda \int_{\Omega}|u|^{k} d x \\
& =\lambda\left(\lambda\|\nabla u\|^{2}+\lambda^{2 \gamma+1}\|\nabla u\|^{2(\gamma+1)}-\lambda^{k-1} \int_{\Omega}|u|^{k} \ln |u| d x-\lambda^{k-1} \ln \lambda \int_{\Omega}|u|^{k} d x\right) \\
& =\lambda j^{\prime}(\lambda) .
\end{aligned}
$$

Thus, $I(\lambda u)>0$ for $0<\lambda<\lambda^{*}, I(\lambda u)<0$ for $\lambda^{*}<\lambda<+\infty$ and $I\left(\lambda^{*} u\right)=0$. The proof is complete.

\section{Lemma 2. [16]}

a) For any function $u \in W_{0}^{1, p}(\Omega)$ such that $\|u\|_{q} \leq B_{q, p}\|\nabla u\|_{p}$, for all $1 \leq q \leq p^{*}$ where $p^{*}=\frac{n p}{n-p}$ if $n>p$ and $p^{*}=\infty$ if $n \leq p$. The best constant $B_{q, p}$ depends only on $\Omega, n, p$ and $q$. We will denote the constant $B_{p, p}$ by $B_{p}$.

b) For any $u \in W_{0}^{1, p}(\Omega), p \geq 1$, and $r \geq 1$, the inequality

$$
\|u\|_{q} \leq C\|\nabla u\|_{p}^{\theta}\|u\|_{r}^{1-\theta}
$$

is valid, where $\theta=\left(\frac{1}{r}-\frac{1}{q}\right)\left(\frac{1}{n}-\frac{1}{p}+\frac{1}{r}\right)^{-1}$ and for $p \geq n=1, r \leq q \leq \infty$; for $n>1$ and $p<n, q \in\left[r, p^{*}\right]$ if $r<p^{*}$ and $q \in\left[p^{*}, r\right]$ if $r \geq p^{*}$ for $p=n>1, r \leq q \leq \infty$; for $p>n>1, r \leq q \leq \infty$.

Here, the constant $C$ depends on $n, p, q$ and $r$.

Lemma 3. [17] Assume that $f: R^{+} \rightarrow R^{+}$be a nonincreasing function and $\sigma$ is a nonnegative constant such that

$$
\int_{t}^{+\infty} f^{1+\sigma}(s) d s \leq \frac{1}{\omega} f^{\sigma}(0) f(t), \quad \forall t \geq 0 .
$$

Hence 
(a) $f(t) \leq f(0) e^{1-\omega t}, \forall t \geq 0$, whenever $\sigma=0$;

(b) $f(t) \leq f(0)\left(\frac{1+\sigma}{1+\omega \sigma t}\right)^{\frac{1}{\sigma}}, \forall t \geq 0$, whenever $\sigma>0$.

\section{Main results}

Now as in [18], we introduce the follows sets:

$$
\begin{aligned}
\mathcal{W}_{1} & =\left\{u \in H_{0}^{1}(\Omega) \backslash\{0\}: J(u)<d\right\}, \\
\mathcal{W}_{2} & =\left\{u \in H_{0}^{1}(\Omega) \backslash\{0\}: J(u)=d\right\}, \\
\mathcal{W} & =\mathcal{W}_{1} \cup \mathcal{W}_{2}, \\
\mathcal{W}_{1}^{+} & =\left\{u \in \mathcal{W}_{1}: I(u)>0\right\}, \\
\mathcal{W}_{2}^{+} & =\left\{u \in \mathcal{W}_{2}: I(u)>0\right\}, \\
\mathcal{W}^{+} & =\mathcal{W}_{1}^{+} \cup \mathcal{W}_{2}^{+}, \\
\mathcal{W}_{1}^{-} & =\left\{u \in \mathcal{W}_{1}: I(u)<0\right\}, \\
\mathcal{W}_{2}^{-} & =\left\{u \in \mathcal{W}_{2}: I(u)<0\right\}, \\
\mathcal{W}^{-} & =\mathcal{W}_{1}^{-} \cup \mathcal{W}_{2}^{-} .
\end{aligned}
$$

Definition 4. (Maximal existence time). Assume that $u$ be weak solutions of problem (1). We define the maximal existence time $T_{\max }$ as follows

$$
T_{\max }=\sup \{T>0: u(t) \text { exists on }[0, T]\} .
$$

Then

(i) If $T_{\max }<\infty$, we see that $u$ blows up in finite time and $T_{\max }$ is the blow-up time;

(ii) If $T_{\max }=\infty$, we see that $u$ is global.

Definition 5. (Weak solution). We define a function $u \in L^{\infty}\left(0, T ; H_{0}^{1}(\Omega)\right)$ with $u_{t} \in L^{2}\left(0, T ; H_{0}^{1}(\Omega)\right)$ to be a weak solution of problem (1) over $[0, T]$, if it satisfies the initial condition $u(0)=u_{0} \in H_{0}^{1}(\Omega) \backslash\{0\}$, and

$$
\begin{aligned}
& <u_{t}, w>+<\nabla u, \nabla w>+<\|\nabla u\|^{2 \gamma} \nabla u, \nabla w>+<\nabla u_{t}, \nabla w> \\
& =\int_{\Omega} u^{k-1} \ln |u| w d x-\int_{\Omega} \oint_{\Omega} u^{k-1} \ln |u| w d s d x,
\end{aligned}
$$

for all $w \in H_{0}^{1}(\Omega)$, and for a.e. $t \in[0, T]$.

Theorem 6. (Global existence). Let $u_{0} \in \mathcal{W}^{+}, 0<J\left(u_{0}\right)<d$ and $I(u)>0$. Hence, there is a unique global weak solution $u$ of (1) satisfying $u(0)=u_{0}$. We obtain $u(t) \in \mathcal{W}^{+}$holds for all $0 \leq t<+\infty$, and the energy estimate

$$
\int_{0}^{t}\left\|u_{s}(s)\right\|_{H_{0}^{1}(\Omega)}^{2} d s+J(u(t)) \leq J\left(u_{0}\right), \quad 0 \leq t \leq+\infty .
$$

Also, the solution decay exponentially provided $u_{0} \in \mathcal{W}_{1}^{+}$.

Proof. (Global existence) In the space $H_{0}^{1}(\Omega)$, we take a Galerkin bases $\left\{w_{j}\right\}_{j=1}^{\infty}$ and define the finite dimensional space

$$
V_{m}=\operatorname{span}\left\{w_{1}, w_{2}, \ldots, w_{m}\right\} .
$$

Let $u_{0 m}$ be an element of $V_{m}$ such that

$$
u_{0 m}=\sum_{j=1}^{m} a_{m j} w_{j} \rightarrow u_{0} \text { strongly in } H_{0}^{1}(\Omega),
$$


as $m \rightarrow \infty$. We can find the approximate solution $u_{m}(x, t)$ of the problem $(1)$ in the form

$$
u_{m}(x, t)=\sum_{j=1}^{m} a_{m j}(t) w_{j}(x)
$$

where the coefficients $a_{m j}(1 \leq j \leq m)$ satisfy the ordinary differential equations

$$
\begin{aligned}
& \int_{\Omega} u_{m t} w_{i} d x+\int_{\Omega} \nabla u_{m} \nabla w_{i} d x+\int_{\Omega}\left\|\nabla u_{m}\right\|^{2 \gamma} \nabla u_{m} \nabla w_{i} d x+\int_{\Omega} \nabla u_{m} \nabla w_{i} d x \\
& \left.=\int_{\Omega}\left|u_{m}\right|^{k-1} \ln \left|u_{m}\right| w_{i} d x-\int_{\Omega} \oint_{\Omega}\left|u_{m}\right|^{k-1} \ln \left|u_{m}\right|\right) w_{i} d s d x
\end{aligned}
$$

for $i \in\{1,2, \ldots, m\}$, with the initial condition

$$
a_{m j}(0)=a_{m j}, \quad j \in\{1,2, \ldots, m\}
$$

Now, multiplying (11) by $a_{m i}^{\prime}$, summing over $i$ from 1 to $m$ and integrating with related to time variable on $[0, t]$, we obtain

$$
\int_{0}^{t}\left\|u_{m s}(s)\right\|_{H_{0}^{1}(\Omega)}^{2} d s+J\left(u_{m}(t)\right) \leq J\left(u_{0 m}\right), \quad 0 \leq t \leq T_{\max }
$$

where $T_{\max }$ is the maximal existence time of solution $u_{m}(t)$. By (9), (13) and the continuity of $J$ that

$$
J\left(u_{m}(0)\right) \rightarrow J\left(u_{0 m}\right), \text { as } m \rightarrow \infty,
$$

with $J\left(u_{0}\right)<d$ and the continuity of functional $J$, by (14), we have

$$
J\left(u_{0 m}\right)<d, \text { for sufficiently large } m \text {. }
$$

And therefore, from (13), we obtain

$$
\int_{0}^{t}\left\|u_{m s}(s)\right\|_{H_{0}^{1}(\Omega)}^{2} d s+J\left(u_{m}(t)\right)<d, \quad 0 \leq t \leq T_{\max }
$$

for sufficiently large $m$. Next, we will show that

$$
u_{m}(t) \in \mathcal{W}_{1}^{+}, \quad t \in\left[0, T_{\max }\right)
$$

for sufficiently large $m$. We suppose that (16) does not hold and think that there exists a smallest time $t_{0}$ such that $u_{m}\left(t_{0}\right) \notin \mathcal{W}_{1}^{+}$. Then, by continuity of $u_{m}\left(t_{0}\right) \in \partial \mathcal{W}_{1}^{+}$. So, we obtain

$$
J\left(u_{m}\left(t_{0}\right)\right)=d
$$

and

$$
I\left(u_{m}\left(t_{0}\right)\right)=0
$$

It is clear that (17) could not occur by (15) while if (18) holds then, by definition of $d$, we get

$$
J\left(u_{m}\left(t_{0}\right)\right) \geq \inf _{u \in \mathcal{N}} J(u)=d,
$$

which contradicts with (15). Thus, we get (16), i.e., $J\left(u_{m}(t)\right)<d$ and $I\left(u_{m}(t)\right)>0$, for any $t \in\left[0, T_{\max }\right)$, for sufficiently large $m$. Hence, by (6), we get

$$
\begin{aligned}
d & >J\left(u_{m}(t)\right) \\
& =\frac{1}{k} I\left(u_{m}(t)\right)+\frac{k-2}{2 k}\left\|\nabla u_{m}(t)\right\|^{2}+\frac{k-2 \gamma-2}{2 k(\gamma+1)}\left\|\nabla u_{m}(t)\right\|^{2(\gamma+1)}+\frac{1}{k^{2}} \int_{\Omega}|u|^{k} d x \\
& \geq \frac{k-2}{2 k}\left\|\nabla u_{m}(t)\right\|^{2}+\frac{\gamma}{2(\gamma+1)}\left\|\nabla u_{m}(t)\right\|^{2(\gamma+1)}+\frac{1}{k^{2}} \int_{\Omega}|u|^{k} d x \geq \frac{k-2}{2 k}\left\|\nabla u_{m}(t)\right\|^{2} .
\end{aligned}
$$


And therefore, we deduce from (15) that

$$
\int_{0}^{t}\left\|u_{m s}(s)\right\|_{H_{0}^{1}(\Omega)}^{2} d s+\frac{k-2}{2 k}\left\|\nabla u_{m}(t)\right\|^{2}<d .
$$

This inequality implies $T_{\max }=+\infty$. Further, by the logarithmic inequality, we get

$$
\begin{aligned}
\left\|\nabla u_{m}(t)\right\|^{2} & =2 J\left(u_{m}(t)\right)+\frac{2}{k} \int_{\Omega}\left|u_{m}(t)\right|^{k} \ln \left|u_{m}(t)\right| d x-\frac{2}{k^{2}}\left\|u_{m}(t)\right\|^{2}-\frac{1}{(\gamma+1)}\left\|\nabla u_{m}(t)\right\|^{2(\gamma+1)} \\
& \leq 2 J\left(u_{m}(0)\right)+\frac{2}{k} \int_{\Omega}\left|u_{m}(t)\right|^{k} \ln \left|u_{m}(t)\right| d x
\end{aligned}
$$

This implies that

$$
\left\|\nabla u_{m}(t)\right\|^{2} \leq 2 J\left(u_{m}(0)\right)+\frac{2}{k} \int_{\Omega}\left|u_{m}(t)\right|^{k} \ln \left|u_{m}(t)\right| d x .
$$

We deduce that

$$
\left\|\nabla u_{m}(t)\right\|^{2} \leq C_{d}, \forall t \in\left[0, T_{\max }\right) .
$$

(Decay estimates) Thanks to $u_{0} \in \mathcal{W}_{1}^{+}$, we deduce from (6) that

$$
\begin{aligned}
J\left(u_{0}\right) & >J(u(t)) \\
& =\frac{1}{k} I(u(t))+\frac{k-2}{2 k}\|\nabla u(t)\|^{2}+\frac{k-2 \gamma-2}{2 k(\gamma+1)}\|\nabla u(t)\|^{2(\gamma+1)}+\frac{1}{k^{2}} \int_{\Omega}|u|^{k} d x, \\
& \geq \frac{k-2}{2 k}\|\nabla u(t)\|^{2}+\frac{k-2 \gamma-2}{2 k(\gamma+1)}\|\nabla u(t)\|^{2(\gamma+1)}+\frac{1}{k^{2}} \int_{\Omega}|u|^{k} d x .
\end{aligned}
$$

By $I(u(t))>0,(7)$ and Lemma 1 , there exists a $\lambda^{*}>1$ such that $I\left(\lambda^{*} u(t)\right)=0$. We have

$$
\begin{aligned}
d & \leq J\left(\lambda^{*} u(t)\right) \\
& =\frac{1}{k} I\left(\lambda^{*} u(t)\right)+\frac{k-2}{2 k}\left\|\nabla\left(\lambda^{*} u(t)\right)\right\|^{2}+\frac{k-2 \gamma-2}{2 k(\gamma+1)}\left\|\nabla\left(\lambda^{*} u(t)\right)\right\|^{2(\gamma+1)}+\frac{1}{k^{2}} \int_{\Omega}\left|\lambda^{*} u(t)\right|^{k} d x \\
& =\frac{k-2}{2 k}\left\|\nabla\left(\lambda^{*} u(t)\right)\right\|^{2}+\frac{k-2 \gamma-2}{2 k(\gamma+1)}\left\|\nabla\left(\lambda^{*} u(t)\right)\right\|^{2(\gamma+1)}+\frac{1}{k^{2}} \int_{\Omega}\left|\lambda^{*} u(t)\right|^{k} d x \\
& =\left(\lambda^{*}\right)^{2}\left(\frac{k-2}{2 k}\right)\|\nabla u(t)\|^{2}+\left(\lambda^{*}\right)^{2(\gamma+1)}\left(\frac{k-2 \gamma-2}{2 k(\gamma+1)}\right)\|\nabla u(t)\|^{2(\gamma+1)}+\left(\lambda^{*}\right)^{k}\left(\frac{1}{k^{2}}\right) \int_{\Omega}|u(t)|^{k} d x \\
& \leq\left(\lambda^{*}\right)^{k}\left(\frac{k-2}{2 k}\|\nabla u(t)\|^{2}+\frac{k-2 \gamma-2}{2 k(\gamma+1)}\|\nabla u(t)\|^{2(\gamma+1)}+\frac{1}{k^{2}} \int_{\Omega}|u(t)|^{k} d x\right) .
\end{aligned}
$$

Using (19) and (20), we have $d \leq\left(\lambda^{*}\right)^{k} J\left(u_{0}\right)$, which implies that

$$
\lambda^{*} \geq\left(\frac{d}{J\left(u_{0}\right)}\right)^{\frac{1}{k}}
$$

By (5), we get

$$
\begin{aligned}
0= & I\left(\lambda^{*} u(t)\right)=\left\|\nabla\left(\lambda^{*} u(t)\right)\right\|^{2}+\left\|\nabla\left(\lambda^{*} u(t)\right)\right\|^{2(\gamma+1)}-\int_{\Omega}\left|\lambda^{*} u(t)\right|^{k} \ln \left|\lambda^{*} u(t)\right| d x \\
= & \left(\lambda^{*}\right)^{2}\|\nabla u(t)\|^{2}+\left(\lambda^{*}\right)^{2(\gamma+1)}\|\nabla u(t)\|^{2(\gamma+1)}-\left(\lambda^{*}\right)^{k} \int_{\Omega}|u(t)|^{k} \ln |u(t)| d x-\left(\lambda^{*}\right)^{k} \ln \left(\lambda^{*}\right) \int_{\Omega}|u(t)|^{k} d x \\
= & \left(\lambda^{*}\right)^{k} I(u(t))+\left(\lambda^{*}\right)^{2}\|\nabla u(t)\|^{2}+\left(\lambda^{*}\right)^{2(\gamma+1)}\|\nabla u(t)\|^{2(\gamma+1)} \\
& -\left(\lambda^{*}\right)^{k}\|\nabla u(t)\|^{2}-\left(\lambda^{*}\right)^{k}\|\nabla u(t)\|^{2(\gamma+1)}-\left(\lambda^{*}\right)^{k} \ln \left(\lambda^{*}\right) \int_{\Omega}|u(t)|^{k} d x \\
= & \left(\lambda^{*}\right)^{k} I(u(t))+\left[\left(\lambda^{*}\right)^{2}-\left(\lambda^{*}\right)^{k}\right]\|\nabla u(t)\|^{2} \\
& +\left[\left(\lambda^{*}\right)^{2(\gamma+1)}-\left(\lambda^{*}\right)^{k}\right]\|\nabla u(t)\|^{2(\gamma+1)}-\left(\lambda^{*}\right)^{k} \ln \left(\lambda^{*}\right) \int_{\Omega}|u(t)|^{k} d x .
\end{aligned}
$$


Using (21) and (22), we have

$$
\begin{aligned}
\left(\lambda^{*}\right)^{k} I(u(t)) & =\left[\left(\lambda^{*}\right)^{k}-\left(\lambda^{*}\right)^{2}\right]\|\nabla u(t)\|^{2}+\left[\left(\lambda^{*}\right)^{k}-\left(\lambda^{*}\right)^{2(\gamma+1)}\right]\|\nabla u(t)\|^{2(\gamma+1)}+\left(\lambda^{*}\right)^{k} \ln \left(\lambda^{*}\right) \int_{\Omega}|u(t)|^{k} d x \\
& \geq\left[\left(\lambda^{*}\right)^{k}-\left(\lambda^{*}\right)^{2}\right] \int_{\Omega}|u(t)|^{k} d x
\end{aligned}
$$

which implies that

$$
I(u(t)) \geq\left[1-\left(\lambda^{*}\right)^{2-k}\right]\|\nabla u(t)\|^{2} .
$$

It follows from (21) and (23) that

$$
\begin{aligned}
I(u(t)) & \geq\left[1-\left(\lambda^{*}\right)^{2-k}\right]\|\nabla u(t)\|^{2} \\
& \geq\left[1-\left(\frac{d}{J\left(u_{0}\right)}\right)^{\frac{2-k}{k}}\right]\|\nabla u(t)\|^{2} \\
& \geq C\left[1-\left(\frac{d}{J\left(u_{0}\right)}\right)^{\frac{2-k}{k}}\right]\|u(t)\|^{2},
\end{aligned}
$$

where $C$ is constant. Hence, by (24), we get

$$
\begin{aligned}
I(u(t)) & \geq \frac{1}{2}\left[1-\left(\frac{d}{J\left(u_{0}\right)}\right)^{\frac{2-k}{k}}\right]\|\nabla u(t)\|^{2}+\frac{C}{2}\left[1-\left(\frac{d}{J\left(u_{0}\right)}\right)^{\frac{2-k}{k}}\right]\|u(t)\|^{2} \\
& \geq C_{1}\left(\|\nabla u(t)\|^{2}+\|u(t)\|^{2}\right) \\
& =C_{1}\|u(t)\|_{H_{0}^{1}(\Omega)}^{2}
\end{aligned}
$$

where

$$
C_{1}=\max \left\{\frac{1}{2}\left[1-\left(\frac{d}{J\left(u_{0}\right)}\right)^{\frac{2-k}{k}}\right], \frac{C}{2}\left[1-\left(\frac{d}{J\left(u_{0}\right)}\right)^{\frac{2-k}{k}}\right]\right\} .
$$

Integrating the $I(u(s))$ with respect to $s$ over $(t, T)$, we get

$$
\begin{aligned}
\int_{t}^{T} I(u(s)) d s & =-\int_{t}^{T} \int_{\Omega} u_{s}(s) u(s) d x d s-\int_{t}^{T} \int_{\Omega} \nabla u_{s}(s) \nabla u(s) d x d s \\
& =\frac{1}{2}\|u(t)\|_{H_{0}^{1}(\Omega)}^{2}-\frac{1}{2}\|u(T)\|_{H_{0}^{1}(\Omega)}^{2} \\
& \leq \frac{1}{2}\|u(t)\|_{H_{0}^{1}(\Omega)}^{2} .
\end{aligned}
$$

From (25) and (26), we have

$$
\int_{t}^{T} C_{1}\|u(t)\|_{H_{0}^{1}(\Omega)}^{2} d s \leq \frac{1}{2}\|u(t)\|_{H_{0}^{1}(\Omega)}^{2} \text { for all } t \in[0, T] .
$$

Let $T \rightarrow+\infty$ in (27), we can have

$$
\int_{t}^{\infty}\|u(t)\|_{H_{0}^{1}(\Omega)}^{2} d s \leq \frac{1}{2 C_{1}}\|u(t)\|_{H_{0}^{1}(\Omega)}^{2} .
$$

From Lemma 3, we get

$$
\|u(t)\|_{H_{0}^{1}(\Omega)}^{2} \leq\|u(0)\|_{H_{0}^{1}(\Omega)}^{2} e^{1-2 C_{1} t} .
$$

The above inequality satisfies that the solution $u$ decays exponentially. 
Theorem 7. (Blow up). Let $u_{0} \in W_{1}^{-}$and assume that $u(t)$ be a unique weak solution to the problem (1). Then $u(t)$ blows up in finite time, that is, there exists $T_{*}>0$ such that

$$
\lim _{t \rightarrow T_{*}}\|u(t)\|_{H_{0}^{1}(\Omega)}^{2}=\infty .
$$

Proof. We show that $u(t)$ blows up at a finite time. Using contradiction, we suppose that $u(t)$ is global. We contract a function $\phi:[0, \infty) \rightarrow \mathbb{R}^{+}$, and

$$
\phi(t)=\int_{0}^{t}\|u(s)\|_{H_{0}^{1}(\Omega)}^{2} d s .
$$

Then, thorough direct calculation, we have

$$
\phi^{\prime}(t)=\|u(s)\|_{H_{0}^{1}(\Omega)}^{2}=2 \int_{0}^{t} \int_{\Omega}\left(u_{s} u+\nabla u_{s} \nabla u\right) d x d s .
$$

By (5) and (29), we have

$$
\begin{aligned}
\phi^{\prime \prime}(t) & =2 \int_{\Omega}\left(u_{s} u+\nabla u_{s} \nabla u\right) d x \\
& =2 \int_{\Omega} u\left(u_{s}-\Delta u_{s}\right) d x \\
& =2 \int_{\Omega}|u|^{k} \ln |u| d x+2 \int_{\Omega} M\left(\|\nabla u\|^{2}\right) u \Delta u d x \\
& =2 \int_{\Omega}|u|^{k} \ln |u| d x-2 \int_{\Omega}\left(1+\|\nabla u\|^{2 \gamma}\right)(\nabla u)^{2} d x \\
& =-2 I(u) .
\end{aligned}
$$

By (30) and $I(u)<0$, we get $\phi^{\prime \prime}(t)>0$, hence

$$
\phi^{\prime}(t)>\phi^{\prime}(0)=\left\|u_{0}\right\|_{H_{0}^{1}(\Omega)}^{2}>0 .
$$

From the Hölder inequality and combining (30), we get

$$
\begin{aligned}
\frac{1}{4}\left(\phi^{\prime}(t)-\phi^{\prime}(0)\right)^{2} & =\frac{1}{4}\left(\int_{0}^{t} \phi^{\prime \prime}(s) d s\right)^{2} \\
& =\left(\int_{0}^{t} \int_{\Omega}\left(u_{s} u+\nabla u_{s} \nabla u\right) d x d s\right)^{2} \\
& \leq \int_{0}^{t}\|u(s)\|_{H_{0}^{1}(\Omega)}^{2} d s \int_{0}^{t}\left\|u_{s}\right\|_{H_{0}^{1}(\Omega)}^{2} d s
\end{aligned}
$$

It follows from (6) and (30) that

$$
\begin{aligned}
\phi^{\prime \prime}(t) & =-2 I(u)=-2 k J(u)+(k-2)\|\nabla u\|^{2}+\frac{k-2 \gamma-2}{\gamma+1}\|\nabla u\|^{2(\gamma+1)}+\frac{2}{k}\|u\|_{k}^{k} \\
& \geq-2 k J\left(u_{0}\right)+2 k \int_{0}^{t}\left\|u_{s}(s)\right\|_{H_{0}^{1}(\Omega)}^{2} d s+(k-2)\|\nabla u\|^{2}+\frac{k-2 \gamma-2}{\gamma+1}\|\nabla u\|^{2(\gamma+1)}+\frac{2}{k}\|u\|_{k}^{k} \\
& \geq 2 k\left(d-J\left(u_{0}\right)\right)+2 k \int_{0}^{t}\left\|u_{s}(s)\right\|_{H_{0}^{1}(\Omega)}^{2} d s .
\end{aligned}
$$

Using (28), (32) and (33), we get

$$
\begin{aligned}
\phi(t) \phi^{\prime \prime}(t) & \geq 2 k \int_{0}^{t}\|u(s)\|_{H_{0}^{1}(\Omega)}^{2} d s \int_{0}^{t}\left\|u_{s}(s)\right\|_{H_{0}^{1}(\Omega)}^{2} d s+2 k\left(d-J\left(u_{0}\right)\right) \phi(t) \\
& \geq 2 k\left(d-J\left(u_{0}\right)\right) \phi(t)+\frac{k}{2}\left(\phi^{\prime}(t)-\phi^{\prime}(0)\right)^{2}
\end{aligned}
$$


By (34), we get

$$
\phi(t) \phi^{\prime \prime}(t)-\frac{k}{2}\left(\phi^{\prime}(t)-\phi^{\prime}(0)\right)^{2} \geq 2 k\left(d-J\left(u_{0}\right)\right)\left\|u_{0}\right\|_{H_{0}^{1}(\Omega)}^{2} t_{0}>0 .
$$

Choose $T>t_{0}$ sufficiently large and let

$$
\psi(t)=\phi(t)+(T-t)\left\|u_{0}\right\|_{H_{0}^{1}(\Omega)}^{2} t_{0}, \forall t \in[0, T] .
$$

Hence, $\mu(t) \geq \phi(t)>0, \mu^{\prime}(t)=\phi^{\prime}(t)-\phi^{\prime}(0)$ and $\mu^{\prime \prime}(t)=\phi^{\prime \prime}(t)>0$, so (35) implies

$$
\mu(t) \mu^{\prime \prime}(t)-\frac{k}{2} \mu^{\prime}(t)^{2} \geq 2 k\left(d-J\left(u_{0}\right)\right)\left\|u_{0}\right\|_{H_{0}^{1}(\Omega)}^{2} t_{0}>0, \text { for all } t \in\left[t_{0}, T\right] .
$$

Let $\psi(t)=\mu(t)^{-\frac{k-2}{2}}$. Thus,

$$
\psi^{\prime}(t)=-\frac{k-2}{2} \mu(t)^{-\frac{k}{2}} \mu^{\prime}(t)
$$

From (36) and (37), we get

$$
\psi^{\prime \prime}(t)=\frac{k-2}{2} \mu(t)^{-\frac{k+2}{2}}\left[\frac{k}{2} \mu^{\prime}(t)^{2}-\mu(t) \mu^{\prime \prime}(t)\right]<0, \text { for all } t \in\left[t_{0}, T\right] .
$$

Therefore, for any sufficiently large $T>t_{0}, \psi(t)$ is a concave function in $\left[t_{0}, T\right]$. Since $\psi\left(t_{0}\right)>0$ and $\psi^{\prime}\left(t_{0}\right)<0$, there exists a finite time $T_{*}$ such that

$$
\lim _{t \rightarrow T_{*}^{-}} \psi(t)=0
$$

Consequently,

$$
\lim _{t \rightarrow T_{*}^{-}} \mu(t)=\infty,
$$

which satisfies

$$
\lim _{t \rightarrow T_{*}^{-}} \int_{0}^{t}\|u(s)\|_{H_{0}^{1}(\Omega)}^{2} d s=\infty
$$

therefore, we have

$$
\lim _{t \rightarrow T_{*}^{-}}\|u(s)\|_{H_{0}^{1}(\Omega)}^{2}=\infty .
$$

This contradicts with $u(t)$ being a global solution.

Author Contributions: All authors contributed equally to the writing of this paper. All authors read and approved the final manuscript.

Conflicts of Interest: "The authors declare no conflict of interest."

\section{Bibliography}

[1] Bialynicki-Birula, I., \& Mycielski, J. (1976). Nonlinear wave mechanics. Annals of Physics, 100(1-2) 62-93.

[2] Gorka, P. (2009). Logarithmic Klein-Gordon equation. Acta Physica Polonica B, 40(1), 59-66.

[3] Chen, H., \& Tian, S. (2015). Initial boundary value problem for a class of semilinear pseudo-parabolic equations with logarithmic nonlinearity. Journal of Differential Equations, 258, 4424-4442.

[4] Chen, H., Luo, P., \& Liu, G. (2015). Global solution and blow-up of a semilinear heat equation with logarithmic nonlinearity. Journal of Mathematical Analysis and Applications, 422(1), 84-98.

[5] Yan, L., \& Yang, Z. (2018). Blow-up and non-extinction for a nonlocal parabolic equation with logarithmic nonlinearity. Boundary Value Problems, 2018, Article No 121. https://doi.org/10.1186/s13661-018-1042-7.

[6] Toualbia, S., Zarai, A., \& Boulaaras, S. (2020). Decay estimate and non-extinction of solutions of p- Laplacian nonlocal heat equations. AIMS Mathematics, 5(3), 1663-1679.

[7] Boulaaras, S., Draifia, A., \& Zennir, K. (2019). General decay of nonlinear viscoelastic Kirchhoff equation with Balakrishnan-Taylor damping and logarithmic nonlinearity. Mathematical Methods in the Applied Sciences, 42, 4795-4814.

[8] Cui, Z., \& Zhang, X. (2020). Blow-up of solutions for a quasilinear parabolic equation with logarithmic nonlinearity term. IAR Journal of Engineering and Technology, 1(3), 72-78. 
[9] Han, Y. (2019). Blow-up at infinity of solutions to a semilinear heat equation with logarithmic nonlinearity. Journal of Mathematical Analysis and Applications, 474, 513-517.

[10] Pişkin, E., Boulaaras, S., \& Irkıl, N. (2021). Qualitative analysis of solutions for the p-Laplacian hyperbolic equation with logarithmic nonlinearity. Mathematical Methods in the Applied Sciences, 44, 5654-4672.

[11] Pişkin, E., \& Cömert, T. (2020). Blow-up of solutions for a parabolic Kirchhoff type equation with logarithmic nonlinearity. Gulf Journal of Mathematics, 9(2), 21-30.

[12] Pişkin, E., \& Irkıl, N. (2019). Well-posedness results for a sixth-order logarithmic Boussinesq equation. FILOMAT, 33(13), 3985-4000.

[13] Pişkin, E., \& Irk1l, N. (In Press). Mathematical behavior of solutions of the Kirchhoff type equation with logarithmic nonlinearity. Boletim da Sociedade Paranaense de Matemática.

[14] Pişkin, E., \& Çalışır, Z. (2020). Decay and blow up at infinite time of solutions for a logarithmic Petrovsky equation. Tbilisi Mathematical Journal, 13(4), 113-127.

[15] Zennir, K., Boulaaras, S., Haiour, M., \& Bayoud, M. (2016). Wave equation with logarithmic nonlinearities in Kirchhoff type. Applied Mathematicsand Information Sciences, 10(6), 2163-2172.

[16] Ladyzhenskaya, O. A., Solonnikov, \& V. A., Uraltseva, N. N. (1988). Linear and Quasi-linear equations of parabolic type. Translations of Mathematical Monographs, 23.

[17] Martinez, P. (1999). A new method to obtain decay rate estimates for dissipative systems. ESAIM: Control, Optimisation and Calculus of Variations, 4, 419-444.

[18] Nhan, L. C., \& Truong, L. X. (2017). Global solution and blow-up for a class of pseudo p-Laplacian evolution equations with logarithmic nonlinearity. Computers and Mathematics with Applications, 73, 2076-2091. 